\title{
Journée Française Maladie de Pompe : une neuvième édition riche de promesses
}

\author{
Sylvie Marion
}

\author{
Le 20 mars dernier, l'Institut de Myologie accueillait des médecins et des chercheurs \\ impliqués sur tout le territoire dans la glycogénose de type 2 pour un événement organisé \\ par le Pr Pascal Laforêt (Garches). Au programme, les progrès les plus récents des \\ connaissances fondamentales, cliniques et thérapeutiques avec cette année une large \\ place accordée aux essais cliniques.
}

\begin{abstract}
I. Maladie de Pompe infantile
Une filière de soins guyano-francilienne

D'après une communication du Dr Samia Pichard (Hôpital Robert Debré, Paris ; Hôpital

d'Argenteuil)

Situé entre le Brésil et le Surinam, la Guyane Française compte près de 250000 habitants. Parmi eux, les Bushinengués (ou Noirs marron) sont les descendants d'esclaves qui se sont installés le long du fleuve Maroni au XIX siècle. Cette communauté représente actuellement 10 à $20 \%$ de la population guyanaise. Elle connait une très forte incidence de la maladie de Pompe avec, sur le plan génétique, deux variants seulement (c.1942G>A p.(Gly648Ser) et c.2560C > T p.(Arg854*) [1]. La glycogénose de type 2 affecte 1 nouveau-né sur 4255 en Guyane Française, mais 1 sur 1604 à Saint Laurent du Maroni. L'incidence de la maladie dans la population guyanaise est probablement sous-estimée. Aucun cas de forme juvénile n'a encore été diagnostiqué. Un essai de dépistage néonatal non obligatoire a été mené entre 2014 et 2015, mais aucun diagnostic positif n'a été porté sur cette période.
\end{abstract}

À l'heure actuelle, si un nourrisson présente des signes évocateurs d'une maladie de Pompe infantile, un diagnostic rapide par dosage de la maltase acide sur goutte de sang séché (papier buvard) est réalisé. Lorsque ce premier examen est positif, un prélèvement sanguin est envoyé à l'hôpital Robert Debré (Paris) pour confirmation diagnostique par test génétique. Si le transfert de l'enfant vers la métropole est possible, l'enzymothérapie de substitution (alglucosidase alfa) est initiée à l'hôpital Robert Debré. L'enfant revient ensuite en Guyane où il poursuit son traitement à l'hôpital de Cayenne.

Depuis 2010, 10 enfants ont été diagnostiqués comme atteints d'une maladie de Pompe infantile. Ils étaient tous Bushinengués, sans notion de consanguinité. Parmi eux, neuf étaient $\mathrm{CRIM}^{1}$ positif, et un seul CRIM négatif. Tous étaient hypotoniques à la naissance. Le premier symptôme de la maladie (détresse respiratoire) est apparu entre l'âge d'un et de cinq mois. L'un des enfants avait une forme trop sévère de la maladie pour être transféré en métropole. Les neuf autres ont pu l'être. Pour tous les enfants, le tableau clinique initial comportait une hépatomégalie, une macroglossie, des fasciculations de la langue et une hypotonie. Des signes de lutte respiratoire ont été observés pour la grande majorité d'entre eux. La plupart (8/9) ne présentait aucun signe d'insuffisance cardiaque, mais tous avaient une cardiomégalie et une hypertrophie myocardique à l'échocardiographie. Une à deux semaines après la confirmation diagnostique, huit patients ont pu débuter l'enzymothérapie substitutive à l'hôpital Robert Debré, sans immunomodulation. La posologie initiale de l'alglucosidase alfa a été de $20 \mathrm{mg} / \mathrm{kg} / 2$ semaines, de $20 \mathrm{mg} / \mathrm{kg} /$ semaine, ou de $40 \mathrm{mg} / \mathrm{kg} / \mathrm{semaine}$ soit quatre fois la dose usuelle pour deux frères dont le second a débuté le traitement à l'âge de deux semaines.

À ce jour, six enfants sont vivants (60\%). Le patient le plus âgé a six ans. Fait déjà connu, le risque de décès s'est avéré plus important pour les enfants dont le diagnostic et donc le traitement ont été les plus tardifs. Le pronostic est lié à la rapidité de prise en charge. Les quatre décès sont survenus aux âges de 7 mois, 8 mois, 15 mois et 2 ans, les trois premiers suite à une insuffisance respiratoire et cardiaque, le dernier suite à une infection. Au dernier bilan, les deux frères traités à $40 \mathrm{mg} / \mathrm{kg} / \mathrm{semaine}$ vont bien. Un seul enfant présente une discrète hypoacousie. Tous ont une alimentation orale normale. Aucun n'a de ventilation non invasive, d'insuffisance cardiaque, de cardiomégalie ni de traitement à visée cardiologique. Tous ont acquis la marche à un âge sensiblement normal (13 à 18 mois). Ils reçoivent l'alglucosidase alfa à la posologie usuelle 
(20 mg/kg/2 semaines) ou deux fois la posologie usuelle. Deux réactions à la perfusion sont survenues, l'une transitoire et l'autre sous immunomodulation.

Ces bons résultats encouragent à sensibiliser et à former les équipes locales à évoquer de principe la maladie de Pompe infantile devant un tableau d'hypotonie néonatale et à orienter très rapidement les enfants vers l'hôpital Robert Debré.

\section{L'essai mini-COMET livre ses tout premiers résultats}

D'après une communication du Pr François

Labarthe (CHRU Clocheville, Tours)

Dans la maladie de Pompe infantile, l'espérance de vie est inférieure à un an en l'absence de traitement. Le premier médicament spécifique mis sur le marché (alglucosidase alfa ou Myozyme ${ }^{\circledR}$ ) améliore la survie, mais il existe un risque d'échappement avec perte d'efficacité à terme (cinq à 10 ans). Diverses hypothèses sont émises pour expliquer ce phénomène : diffusion modeste du produit dans les muscles, immunogénicité, apparition d'une atteinte du système nerveux central... Le néoGAA ou avalglucosidase alfa est une enzymothérapie de nouvelle génération. Elle améliorerait l'adressage et l'entrée de l'enzyme dans les cellules musculaires grâce à l'ajout en surface de bismannose-6-phosphate.

Un essai clinique de phase 2 multicentrique international, randomisé, en ouvert appelé "miniCOMET " ${ }^{2}$ a été lancé voici un peu plus d'un an. La première inclusion a été réalisée en octobre 2017. Son objectif est d'évaluer la sécurité d'emploi, la tolérance, la pharmacocinétique et l'efficacité du néoGAA administré une semaine sur deux à 20 enfants atteints d'une forme classique de maladie de Pompe infantile (avec notamment cardiomyopathie au moment du diagnostic) et traités auparavant par Myozyme ${ }^{\circledast}$ depuis au moins six mois, avec réponse suboptimale ou déclin malgré le traitement. Un taux élevé d'anticorps anti-alglucosidase alfa ( $\geq 1 / 25600$ ) constitue un critère d'exclusion. La France a inclus quatre patients sur deux sites: le CHRU Clocheville (Tours) et l'hôpital Robert Debré (Paris).

L'essai comporte trois cohortes, initiées de façon séquentielle. La première rassemble six enfants qui ont connu un déclin malgré le traitement par Myozyme $^{\circledast}$. Dans le cadre de l'essai mini-COMET, ils reçoivent le néoGAA à la dose de $20 \mathrm{mg} / \mathrm{kg} /$ 2 semaines pendant six mois ( 13 doses au total). Quatre patients ont déjà terminé le semestre de traitement et sont passés en phase d'extension. La deuxième cohorte inclut cinq enfants, qui ont également connu un déclin malgré l'enzymothérapie par Myozyme $e^{\circledast}$. Ils sont traités par néoGAA à la posologie de $40 \mathrm{mg} / \mathrm{kg} / 2$ semaines pendant six mois. La troisième cohorte concerne 10 enfants chez lesquels l'efficacité du Myozyme ${ }^{\circledast}$ n'a été que partielle. Répartie en deux groupes, le traitement a démarré en décembre 2018 : l'un reçoit pendant six mois du Myozyme ${ }^{\circledast}$, l'autre pendant six mois du néoGAA à la posologie la plus forte la mieux tolérée dans les étapes précédentes de l'essai soit $40 \mathrm{mg} /$ $\mathrm{kg} / 2$ semaines. Une phase d'extension est d'ores et déjà prévue.

Les critères primaires de l'essai mini-COMET sont en lien avec la sécurité d'emploi et la tolérance du néoGAA (événements indésirables et immunogénicité). À ce stade, aucune donnée d'efficacité ni de pharmacocinétique n'est encore disponible, mais des résultats préliminaires ont été présentés lors du dernier WORLD $D^{3}$ Symposium à Orlando. Ils concernent les deux premières cohortes et donc des patients atteints d'une forme sévère, avec début de l'enzymothérapie très tôt dans la vie. Leur âge moyen est de 7,5 ans pour la cohorte 1,8 ans pour la cohorte 2. La tolérance du néoGAA a été bonne. Tous les enfants ont présenté des effets secondaires mais relativement modestes et pas forcément en lien avec le traitement. Aucun patient n'a développé d'anticorps anti-avalglucosidase alfa à six mois. Aucun n'avait reçu au préalable de traitement immunosuppresseur. À noter que certains patients avaient à l'inclusion des taux modérés d'anticorps anti-alglucosidase alfa, qui ont disparu par la suite.

\section{Maladie de Pompe chez l'adulte Expérience du Myozyme ${ }^{\circledast}$ en Grèce : plus de 10 ans déjà}

D'après une communication du Dr Constantinos Papadopoulos (Hôpital universitaire

neurologique Eginitio, Athènes)

Les premiers patients traités en Grèce par alglucosidase alfa (Myozyme ${ }^{\circledast}$ ) étaient deux sœurs, dans le cadre du programme d'accès anticipé ${ }^{4}$ dès février 2006. Elles sont atteintes d'une forme juvénile de la maladie de Pompe et toutes deux homozygotes. Depuis, 12 autres patients moins sévères ont commencé à recevoir le même traitement, également à la dose de $20 \mathrm{mg} / \mathrm{kg} / 2$ semaines et avec une évaluation annuelle. Ces 14 patients atteints d'une forme tardive de la maladie de Pompe étaient âgés de 13 à 72 ans à la mise au traitement par Myozyme $^{\circledast}$. L'évolution des cinq premiers patients mis sous enzymothérapie substitutive a fait l'objet d'une publication [2]. Un registre grec sur la maladie de Pompe est en cours de constitution.

À ce jour, les 14 patients ont reçu des perfusions d'alglucosidase alfa pendant 9 à 136 mois. Au 
moment de l'instauration du traitement, un patient était en fauteuil roulant et un autre marchait avec un aide unilatérale, un patient était sous ventilation invasive et quatre sous ventilation non invasive nocturne. L'enzymothérapie a entrainé une amélioration les deux premières années chez tous les patients, pour l'ensemble des paramètres (capacité vitale forcée ou CVF, force musculaire, performances motrices). Leurs résultats se sont ensuite détériorés. L'effet de l'enzymothérapie est donc maximal les deux premières années, mais il est de courte durée avec reprise du déclin dans un second temps. L'âge de début de la maladie influence la réponse au traitement. Une maladie de Pompe tardive qui commence avant l'adolescence se traduit par de moins bons résultats en termes de force musculaire et de CVF en position debout. De même, le délai entre le début des symptômes et le début de l'enzymothérapie de substitution influence la réponse thérapeutique. Le Myozyme $^{\circledR}$ a été bien toléré par l'ensemble des patients de la cohorte grecque, avec seulement quelques symptômes mineurs lors de la première perfusion (céphalées, prurit). Un taux très élevé d'anticorps anti-alglucosidase alfa a été retrouvé chez un patient. Les 13 autres ont connu une augmentation transitoire de ce taux les six premiers mois de traitement. Cinq patients ont arrêté l'enzymothérapie, après un délai d'un à cinq ans: trois pour une absence de bénéfice perçu, un après une fracture de hanche, et le cinquième pour un taux élevé d'anticorps antialglucosidase alfa mais la reprise de son traitement est programmée. La notion d'absence de bénéfice est subjective. Bien expliquer au patient ce qu'il peut attendre de l'enzymothérapie aide à réduire le risque d'arrêt ultérieur.

La nécessité d'améliorer l'efficacité du traitement dans la forme tardive de la maladie a conduit à réaliser deux études sur l'exercice physique, dont on sait qu'il empêche le déconditionnement et l'atrophie musculaire. La première a rassemblé cinq patients et comportait un programme d'exercice physique supervisé, trois fois par semaine pendant 20 semaines, à domicile. Ses résultats montrent une amélioration des performances au test de 6 minutes de marche et de la force musculaire, sans changement significatif de la masse maigre totale et des membres inférieurs [3]. La deuxième étude a exploré l'effet de l'exercice physique pratiqué cette fois pendant la perfusion de Myozyme ${ }^{\circledR}$, pour augmenter le flux sanguin musculaire. L'efficacité de l'enzymothérapie n'a pas été améliorée [4].

Une dernière étude menée sur la cohorte grecque a exploré la composition corporelle par absorptiométrie biphotonique à rayons $\mathrm{X}$ (DXA). Sur les neuf patients inclus, deux avaient initialement une ostéopénie, trois une diminution de la densité minérale osseuse en L2-L4 et six une diminution de la densité minérale osseuse du col fémoral. La majorité présentait une augmentation de la masse grasse. L'enzymothérapie substitutive n'a pas eu d'impact sur la composition corporelle [5].

\section{Le registre Français de la maladie de Pompe en 2019}

D'après une communication du Pr Pascal Laforêt (Hôpital Raymond Poincaré, Garches)

Créé en 2004, le registre Français de la maladie de Pompe a pour objectif de recenser tous les patients atteints de glycogénose de type 2 en France dans un but de recherche clinique et fondamentale : épidémiologie, profils moléculaires, histoire naturelle (grâce aux patients non traités par enzymothérapie substitutive), effets des traitements, causes de décès... Au premier février 2019, il comptait 212 patients, soit 198 adultes et 14 enfants. Ils sont 70 à être suivis à Paris et dans sa région, 32 à Lyon, 15 à Lille, 14 à Marseille et 13 à Nancy. Une dizaine de nouveaux patients adultes sont inclus chaque année dans le registre, ce qui correspond peu ou prou au nombre de nouveaux diagnostics annuels en France. Il y a donc une quasi exhaustivité des données pour les patients adultes. Ce n'est pas encore le cas pour les enfants, dont les inclusions devraient monter en puissance en 2019.

Sur l'ensemble des patients du registre, 153 sont traités par alglucosidase alfa. Les patients non traités par enzymothérapie représentent donc près du quart du registre, une proportion loin d'être négligeable. Certains sont cependant inclus dans des essais cliniques, à l'exemple de l'étude COMET. Parmi les 14 enfants inscrits au registre, 10 sont traités par alglucosidase alfa.

Le registre Français permet chaque année différents travaux de recherche. Une étude sur l'épidémiologie et les caractéristiques génétiques des patients atteints d'une maladie de Pompe à début tardif est ainsi parue fin 2018 [6]. Une autre étude, en cours de révision avant publication, porte sur le devenir à long terme des patients traités par enzymothérapie. Elle confirme la grande variabilité interindividuelle de la réponse thérapeutique. À quatre ans de traitement, les patients semblent néanmoins globalement stabilisés sur le plan moteur et respiratoire. Le nombre de patients sous ventilation non invasive (VNI) a un peu augmenté depuis leur mise sous traitement, mais pas celui des patients sous ventilation invasive (VI). La pente de déclin de la capacité vitale (CV) sous traitement reste moins importante que celle de l'histoire naturelle. Cependant, une comparaison stricto sensu n'est pas possible, comme elle 
le serait dans le cadre d'un essai clinique comparant une cohorte traitée par alglucosidase alfa et une autre qui ne l'est pas. En ce qui concerne le test de 6 minutes de marche (6 MWT), le traitement entraine une amélioration durant les trois premières années, suivie d'une dégradation. Là encore, le déclin est probablement ralenti mais le manque de données d'histoire naturelle pour ce test complique la comparaison. Les meilleurs résultats pour le 6 MWT sous traitement sont obtenus lorsque la maladie évolue depuis moins de 15 ans.

Une autre étude récente a porté sur la mortalité sous alglucosidase alfa. Quinze patients traités sont décédés, le plus souvent d'une cause respiratoire et ce même en l'absence d'atteinte respiratoire sévère. Certains patients décédés de cause respiratoire n'étaient pas sous VI mais sous VNI. Aucun décès n'est consécutif aux effets secondaires de l'enzymothérapie substitutive. Sa durée au moment du décès était de 11 à 122 mois. Parmi les patients décédés, deux ont fait une mort subite sans plus de précision, et notamment sans information sur une éventuelle atteinte cardiaque.

Toujours à partir des données du registre, d'autres publications sont en projet, notamment sur les patients en interruption d'enzymothérapie, les événements indésirables du traitement, le tétraglucose (Glc4), l'échelle R-PAct et les patients non traités par alglucosidase alfa.

\section{R-PAct à l'épreuve du registre français de la maladie de Pompe \\ D'après une communication du Dr Claire Lefeuure (Hôpital Raymond Poincaré, Garches)}

Conçue par une équipe néerlandaise, l'échelle Rasch-built Pompe-specific activity (R-PAct) a été développée de façon spécifique pour la glycogénose de type 2 afin de mesurer les effets de la maladie sur la capacité des patients à effectuer les activités de la vie quotidienne et sur leur participation à la vie sociale [7]. Pour construire ce nouvel outil, un modèle mathématique a été créé afin d'identifier les items pertinents et déterminants d'évaluation des impacts de la maladie et de son traitement. L'équipe s'est appuyé sur une cohorte de 186 patients de sept pays, âgés de plus de 16 ans (âge moyen: 50 ans) avec une durée médiane d'évolution de la maladie de 11 ans. Parmi eux, $37 \%$ étaient ambulants sans aide, $16 \%$ utilisaient une aide technique, $47 \%$ un fauteuil roulant. Près de la moitié (44\%) étaient sous ventilation mécanique. Une série initiale de 136 items fonctionnels et occupationnels a été soumise à une analyse statistique (modèle de Rasch) qui a permis d'élaborer un questionnaire de 18 items: se peigner les cheveux, prendre une douche, marcher vite, etc. Pour chaque item, le patient a trois choix possibles : non ( 0 point), oui avec difficultés (1 point) et oui sans difficulté ( 2 points). Le score final va donc de 0 à 36 points. L'échelle R-PAct a été utilisée sur une cohorte de 65 patients du registre français de la maladie de Pompe, de 56 ans d'âge moyen. Plus de la moitié (55\%) étaient ambulants sans aide, $34 \%$ avec une aide technique, $11 \%$ se déplaçaient en fauteuil roulant. L'analyse a retrouvé une bonne corrélation de l'échelle R-PAct avec les données relatives à la marche et à l'autonomie de marche mesurées par d'autres tests $(6$ minutes de marche, 4 marches, échelle de Vignos). Il existe également une bonne corrélation avec le recours aux appareils de ventilation, mais pas avec la capacité vitale. Souple et spécifique, ce nouvel outil pourrait à l'avenir être notamment utilisé de façon étendue dans le registre Français de la maladie de Pompe.

\section{Arrêt de l'enzymothérapie chez l'adulte: des causes et des conséquences mieux connues \\ D'après une communication du Dr Emmanuelle Salort-Campana (CHU La Timone, Marseille)}

Sur une période de 14 ans, 145 patients du registre français de la maladie de Pompe ont été traités par alglucosidase alfa. Ils ont été 22 à arrêter ce traitement après un délai de 1 à 84 mois. Leur âge moyen au moment du diagnostic était de 47,8 ans. Et l'intervalle entre diagnostic et début de l'enzymothérapie, de 1 à 24 ans. La première cause d'arrêt est la décision du patient. Ce premier groupe (G1) rassemble 10 patients qui ont choisi d'interrompre l'enzymothérapie en raison de la fatigue qu'elle engendre, de la perception d'une progression de la maladie en dépit des perfusions ou d'une non-compliance (parfois pour raison professionnelle). Les effets indésirables constituent la deuxième cause d'arrêt. Ce deuxième groupe (G2) compte 12 patients, en majorité de femmes mais la différence n'est pas significative, qui ont connu des réactions à la perfusion (RAP) modérée à sévère.

Sur les 22 patients qui ont arrêté le traitement, sept sont décédés à ce jour soit $31,8 \%$, une mortalité supérieure à celle enregistrée dans l'ensemble du registre (13,3\%). L'arrêt de l'enzymothérapie substitutive entraine donc une surmortalité. L'âge moyen au moment du décès atteint 64 ans. Il survient après une durée médiane de cessation du traitement de 38 mois. Cinq des 10 patients du G1 sont décédés (50\%), versus 2/12 dans le G2. Deux décès sont dus à une insuffisance respiratoire, un à une pneumopathie de déglutition, un à une cause cardiorespiratoire, un à un cancer du pancréas, un dans un 
contexte de syndrome douloureux abdominal et le dernier est survenu à domicile de cause inconnue. Les patients décédés étaient en moyenne plus âgés au début et à la fin du traitement que ceux qui ne sont pas décédés.

Les conséquences de l'arrêt du traitement sur les fonctions respiratoire et motrice s'avèrent variables selon les patients. Parmi ceux dont l'interruption thérapeutique a duré plus de 24 mois, les performances au test de 6 minutes de marche (6 MWT) ont chuté pour quatre patients, mais elles ont augmenté ou se sont stabilisées pour les autres. Lorsque l'arrêt du traitement a duré moins de 24 mois, quatre patients ont eu des résultats stables au 6 MWT, alors qu'ils ont baissé chez les autres. En ce qui concerne les performances respiratoires, plus l'arrêt de l'enzymothérapie est prolongé, plus la capacité vitale $(\mathrm{CV})$ baisse. Le risque d'avoir recours à une aide à la marche et à une augmentation de l'assistance respiratoire augmente avec la durée de cessation de l'enzymothérapie substitutive. Son interruption à long terme semble donc être associée à une aggravation respiratoire et ambulatoire.

Parmi les 22 patients qui ont arrêté leur traitement, cinq l'ont ensuite repris et, parmi eux, deux l'ont de nouveau arrêté. Dans la littérature, plusieurs publications font état des effets d'un arrêt puis d'une reprise du traitement. La plus grande étude, réalisée en Suisse, ne concerne que sept patients. Dans ce pays, le remboursement du Myozyme ${ }^{\circledR}$ a cessé de façon temporaire, entrainant de facto une interruption de traitement pour sept patients, puis sa reprise. Leurs résultats au 6 MWT ont chuté après l'arrêt du traitement, pour s'améliorer lorsqu'il a pu reprendre, sans toutefois revenir à l'état antérieur. De même, leur CV s'est améliorée ou stabilisée à la reprise thérapeutique, sans revenir à l'état antérieur [8]. Une étude menée au Royaume-Uni fait état de résultats similaires [9].

Tout doit donc être fait pour éviter une interruption thérapeutique. Quand elle survient néanmoins, le suivi doit se poursuivre. Un patient qui arrête son traitement doit continuer d'être évalué à intervalles réguliers, peut-être même davantage que les autres. En amont, sachant que les contraintes qu'entrainent les perfusions sont des causes majeures de décision d'arrêt par le patient, l'éducation thérapeutique prend tout son sens pour améliorer l'adhésion thérapeutique. En France, l'enzymothérapie substitutive à domicile n'est pas encore une réalité, en particulier par crainte des RAP. Néanmoins, une prise en charge dans un hôpital de proximité s'avère parfois possible.

\section{Recherche}

Les cellules satellites musculaires sont fonctionnelles mais échouent à réparer le muscle

D'après une communication du Dr Karl Rouger (INRA, UMR 703, ONIRIS, Atlantic Gene

Therapies, Nantes)

La glycogénose de type 2 entraine des modifications des fibres musculaires (vacuolisation, accumulation d'agrégats autophagiques...). Ces événements n'entrainent pourtant ni nécrose massive, ni régénération des fibres musculaires. La sévérité des dommages engendrés par la maladie de Pompe n'est pas associée à une réparation par les cellules satellites musculaires. Cette thématique a été le sujet de la thèse de biologie de Lydie Lagalice ${ }^{5}$, soutenue en octobre 2018.

Dans un premier temps, elle a cherché à évaluer l'incidence des modifications tissulaires de la maladie de Pompe sur le comportement des cellules satellites, grâce à une étude histo-pathologique longitudinale menée sur un modèle murin de la maladie (souris). Une surcharge en glycogène apparaît dans les muscles après l'âge de 2,5 mois. Elle reste stable ensuite, touchant 8 à $10 \%$ des fibres musculaires. Il se produit également une augmentation progressive des cellules contenant des agrégats liés à l'autophagie et du nombre de ces mêmes agrégats. La vacuolisation progressive des cellules musculaires est corrélée à l'agrégation autophagique. Il existe en revanche très peu de fibres nécrotiques, quel que soit l'âge de la souris. De même, la régénération est extrêmement rare. Il se produit par ailleurs une augmentation progressive du nombre de fibres musculaires avec un noyau internalisé. La souris modèle de la maladie de Pompe possède bien un stock de cellules satellites musculaires, identique à tous les âges et comparable à celui de la souris sauvage. Ces cellules conservent leurs capacités à s'activer et à s'impliquer dans le processus myogénique, mais leur activation diminue en dépit de l'augmentation des dommages structuraux.

Dans un deuxième temps, le potentiel intrinsèque des cellules satellites musculaires a été étudié. Leur morphologie ainsi que leurs capacités proliférative et de différenciation se sont avérées identiques chez la souris modèle de la maladie et chez la souris sauvage. Les cellules satellites musculaires semblent donc fonctionnelles dans la glycogénose de type 2 . Après induction d'une lésion (injection de cardiotoxine), l'analyse histologique des muscles des souris modèles de la maladie retrouve, à 21 jours, une taille et une distribution des fibres musculaires presque identiques à celles des souris sauvages. Les cellules 
satellites musculaires sont donc capables de s'activer et de contribuer à la régénération musculaire. Elles restent intrinsèquement fonctionnelles. Alors pourquoi leur activation diminue-t-elle spontanément au fil des dommages musculaires dans la maladie de Pompe? Le signal d'activation (trop faible, mal transmis) ou sa perception par les cellules satellites pourrait être en cause [10].

\section{Forme tardive : analyser les biopsies musculaires pour éclairer \\ la physiopathologie \\ D'après une communication du Dr Edoardo Malfatti (Hôpital Raymond Poincaré, Garches)}

L'accumulation de glycogène caractéristique de la glycogénose de type 2 est objectivable à la coloration au $\mathrm{PAS}^{6}$, mais aussi en microscopie électronique. Dans 70 à $89 \%$ des cas de maladie de Pompe à début tardif (late onset Pompe disease ou LOPD), l'examen de la biopsie musculaire retrouve des anomalies: vacuoles (parfois optiquement vides), activation lysosomale, accumulation d'agrégats autophagiques... Une étude a été menée, en aveugle, sur 16 patients du registre français de la maladie de Pompe atteints de LOPD et non traités par enzymothérapie au moment de leur biopsie musculaire. Ses résultats ne sont pas encore publiés. L'âge moyen de ces 16 patients $(9$ hommes, 7 femmes) était de 45 ans. Après analyse en microscopie optique, leurs biopsies ont pu être réparties en quatre groupes histologiques. Dans le premier (3 patients), la maladie est très sévère sur le plan histologique, avec vacuolisation de nombreuses fibres musculaires, activation lysosomale massive et forte variation de la taille des fibres musculaires, sans signe de nécrose ni de régénération. Dans le deuxième groupe (5 patients), les anomalies histologiques sont plus discrètes, avec quelques fibres musculaires atrophiques contenant des vacuoles, une surcharge glycogénique des vacuoles et du cytoplasme. Une activation lysosomale est retrouvée dans les fibres sans vacuoles. Elle pourrait donc être le premier phénomène survenant dans la maladie de Pompe. Le troisième groupe (5 patients) se caractérise par une myopathie vacuolaire minime, et le quatrième ( 3 patients) par une absence d'anomalies. Les mêmes biopsies ont été analysées en microscopie électronique. Elle a révélé une autophagie prédominante dans le premier groupe, la présence de glycogène et de quelques agrégats de matériel autophagique dans le deuxième, la présence de glycogène à l'intérieur des lysosomes et du matériel autophagique dans le troisième, la présence de quelques

agrégats autophagiques parfois associés à du glycogène à l'intérieur des lysosomes dans le quatrième (pourtant exempt d'anomalie en microscopie optique).

Cette étude permet de conclure que le spectre histologique s'avère très variable dans la maladie de Pompe, avec des patients quasi asymptomatiques sur ce plan. L'augmentation de l'autophagie pourrait être le mécanisme initial, mais ce point nécessite des travaux complémentaires. Le projet French MorphoPompePhagy, porté par Edoardo Malfatti et Pascal Laforêt, va désormais s'attacher à étendre l'analyse des biopsies musculaires à un plus grand nombre de patients, traités ou non par enzymothérapie substitutive.

\section{Signes ophtalmologiques : les leçons de la littérature \\ D'après une communication du Dr Tuy Nga Brignol (AFM-Téléthon, Éury)}

Des informations limitées sont disponibles sur les manifestations oculaires dans la maladie de Pompe. Chez l'enfant, il s'agit essentiellement de strabisme et de ptosis bilatéral [11-13]. Des observations en microscopie de fotus provenant d'avortements (spontanés ou thérapeutiques) liés à la glycogénose de type 2, d'autopsies ou de muscles oculaires réséqués lors de la chirurgie du strabisme chez des enfants atteints ont été rapportées. La première étude histo-pathologique de l'œil en microscopie optique a été réalisée en 1965, chez un nourrisson de sexe féminin décédée à l'âge de 5 mois [14]. Elle montre que des dépôts de glycogène sont présents dans pratiquement tous les tissus oculaires, à l'exception de l'épithélium pigmentaire de la rétine. De ce fait, un suivi ophtalmologique dans l'évolution à moyen - long terme de la maladie de Pompe chez l'enfant est nécessaire. La prise en charge doit inclure des bilans ophtalmologiques complets, pour dépister de façon précoce des atteintes oculaires potentiellement amblyogéniques.

Une collaboration entre les Centres de référence et de compétences des maladies neuromusculaires rares du réseau FILNEMUS (www.filnemus.fr), de la filière des maladies métaboliques G2M (www.filiere$\mathrm{g} 2 \mathrm{~m}$.fr) et de la filière des maladies rares sensorielles SENSGENE (www.sensgene.com) serait justifiée, afin de mieux définir le spectre des anomalies oculaires susceptibles de survenir avec l'amélioration de la survie. De nouvelles études sont également nécessaires pour mieux comprendre les atteintes oculaires dans la maladie de Pompe, ainsi que les améliorations possibles liées à l'enzymothérapie substitutive ou à d'autres thérapies émergentes.
NOTES

${ }^{6}$ Periodic Acid Schiff. 
IV. Essais cliniques COMET compare le néoGAA au Myozyme

D'après une communication du Pr Sabrina Sacconi (CHU de Nice)

Enzyme recombinante de nouvelle génération, l'avalglucosidase alfa ou néoGAA a été développée par le laboratoire Sanofi Genzyme à partir de l'alglucosidase alfa $\left(\right.$ Myozyme $\left.^{\circledR}\right)$ à laquelle a été ajouté du mannose-6-phosphate pour en améliorer l'internalisation dans les cellules musculaires et les lysosomes. Initié fin 2016, COMET est un essai clinique de phase III, international multicentrique, contrôlé en double aveugle, destiné à évaluer l'efficacité, la sécurité et la tolérance du néoGAA versus Myo$z y m e^{\circledast}$. Il s'agit de démontrer la supériorité, ou la non-infériorité, du néoGAA. Les patients inclus doivent être atteints d'une forme tardive de la maladie de Pompe, porteurs de deux mutations pathogènes sur le gène $G A A$, âgés de trois ans ou plus et naïfs de tout traitement. Ils doivent être capables de marcher plus de 40 mètres sans aide, et leur capacité vitale forcée (CVF) en position assise doit être comprise entre 30 et $85 \%$ de la valeur prédite. Le traitement est administré par voie intraveineuse à la posologie de $20 \mathrm{mg} / \mathrm{kg} / 2$ semaines dans les deux groupes, Myozyme ${ }^{\circledast}$ et néoGAA, pendant 12 mois (phase de double aveugle), suivi de 24 mois où tous les patients reçoivent un traitement par néoGAA. Le critère principal de l'essai est l'évolution de la CVF en position assise en pourcentage de la valeur prédite. L'objectif d'inclusion (96 patients) sera atteint le 29/03/2019.

Les centres investigateurs sont situés aux États-Unis (six sites et 31 patients inclus), en Australie, en Asie, en Europe (17 sites). Pays majeur de cet essai, la France compte 10 centres investigateurs qui vont recruter 10 à 12 patients sur 96 . La fin de la période double aveugle est prévue pour mars 2020, et ses résultats préliminaires attendus pour le dernier trimestre 2020.

Un essai de phase III pour une nouvelle GAA avec molécule chaperonne

D'après une communication du Dr Françoise Bouhour (Hôpital Pierre Wertheimer, CHU

de Lyon)

Le laboratoire Amicus Therapeutics explore l'association d'une enzyme de nouvelle génération (ATB200) associée à une molécule chaperonne (miglustat ou AT2221). L'ATB200 comporte davantage de mannose 6-phosphate à sa surface que l'alglucosidase alfa. Le miglustat se fixe sur l'ATB200 avec une grande affinité, car sa conformation est proche de celle du glycogène, substrat de l'alpha-glucosidase acide (GAA). Il stabilise l'enzyme en milieu alcalin, comme le sang. Dans la circulation sanguine, l'ATB200 garderait donc sa conformation et pourrait ainsi à la fois pénétrer en plus grande quantité en intracellulaire et en intralysosomal, et avoir une activité préservée.

Un essai clinique international de phase $\mathrm{I} / \mathrm{II}^{7}$ a déjà exploré l'administration conjointe d'ATB200 (par voie intraveineuse) et d'AT2221 (voie orale) sur quatre cohortes de patients adultes atteints d'une maladie de Pompe. Chez 11 patients ambulants déjà sous enzymothérapie de substitution (ERTSwitch) et cinq patients naïfs d'enzymothérapie de substitution (ERT-Naive), les résultats au test de 6 minutes de marche (6MWT) ont augmenté de 50 mètres après près de 2 ans de traitement, avec amélioration également des autres scores fonctionnels et de la force musculaire. La fonction respiratoire des patients ERT-Naive s'est également discrètement améliorée. Elle s'est stabilisée chez les patients déjà sous enzymothérapie de substitution. L'association ATB200/AT2221 a été bien tolérée, avec des évènements indésirables en général modérés, transitoires et peu fréquents $(1,3 \%$ ) après un total de plus de 1110 perfusions. Un seul traitement a dû être arrêté en raison d'une réaction à la perfusion.

Ces bons résultats ont conduit au lancement d'un essai de phase III $^{8}$, randomisé en double aveugle. Il prévoit d'inclure 100 à 110 patients âgés de plus de 18 ans atteints d'une maladie de Pompe à début tardif confirmée, naïfs d'enzymothérapie substitutive $(\mathrm{n}=30)$ ou sous enzymothérapie depuis plus de deux ans, d'un poids supérieur à $40 \mathrm{~kg}$ et dont la capacité vitale forcée se situe entre 30 et $80 \%$. L'utilisation d'une ventilation invasive ou non invasive plus de 6 heures dans la journée constitue un critère d'exclusion. Les participants receuront durant 52 semaines soit ATB200/AT2221, soit alglucosidase/placebo. Le critère primaire d'évaluation est l'évolution du 6 MWT. Cet essai va rassembler 87 centres investigateurs dans le monde, dont cinq en France à Lyon, Marseille, Nice, Paris et Lille. Les inclusions débuteront à partir d'octobre 2019.

Thérapie génique : après la souris et le primate, bientôt un essai clinique D'après une communication du Dr Giuseppe Ronzitti (Généthon, INSERM U951

INTEGRARE, Éury)

Dans la maladie de Pompe, l'enzymothérapie substitutive connaît plusieurs limitations à commencer par son immunogénicité, son effet limité chez les patients $\mathrm{CRIM}^{9}$ négatif et son coût. Généthon travaille depuis plusieurs années au développement d'une alternative thérapeutique: la thérapie 
génique. Elle utilise comme vecteur un virus adénoassocié (adeno-associated virus ou AAV). La stratégie choisie a été de cibler, non pas l'ensemble des muscles, mais le foie. L'AAV porteur d'un gène GAA modifié pénètre dans le noyau des hépatocytes qui secrètent dès lors l'enzyme alpha-glucosidase alpha (GAA). Une fois libérée dans la circulation sanguine, cette GAA secrétable devrait être mieux tolérée sur le plan immunitaire qu'une enzyme exogène.

Chez la souris modèle de la maladie de Pompe, cette thérapie réduit bien la taille des lysosomes et modifie le phénotype: amélioration des fonctions cardiaque, musculaire et respiratoire, prolongation de la survie de plusieurs mois. Elle a été comparée à une enzymothérapie par alglucosidase alfa toujours sur une souris modèle de la maladie. L'alglucosidase alfa à la dose de $20 \mathrm{mg} / \mathrm{kg}$ n'a pas entrainé d'amélioration de la force musculaire, ni de réduction de l'accumulation de glycogène dans les muscles et le système nerveux central. La thérapie génique s'est montrée plus efficace, à la dose de $10^{11} \mathrm{vg} / \mathrm{kg}$, avec notamment une réduction du glycogène cérébral (jusqu'à $50 \%$ ) [15]. De même, chez le macaque rhésus, elle a augmenté la production et l'activité de la GAA dans le plasma, sans évènement indésirable jusqu'à une dose de $2 \times 10^{13} \mathrm{vg} / \mathrm{kg}$. Le développement clinique de ce produit (SPK-3006) est désormais dans les mains du laboratoire Sparks Therapeutics, qui envisage de déposer une demande d'autorisation d'essai clinique dès 2019 au ÉtatsUnis, puis en Europe.

The French Pompe Disease Day: a $9^{\text {th }}$ edition full of promises

Information de l'Association Francophone des Glycogénoses

\section{- Une mascotte voyageuse}

Le projet international Hope a pour objectif de sensibiliser à la maladie de Pompe. Il est incarné par une mascotte (un paresseux) qui rend visite aux familles concernées dans différents pays, pour leur apporter joie et espoir. Son tour du monde a commencé en 2017 au Royaume-Uni. Hope est arrivé en France en novembre 2018. Il participera à la $19^{e}$ édition de la course cycliste Paris - Brest - Paris avec plusieurs membres de la Malaysia Lysosomal Disease Association, en août 2019. Ses aventures sont partagés sur les réseaux sociaux (groupe Facebook Hope Travels - Pompe Awareness notamment).

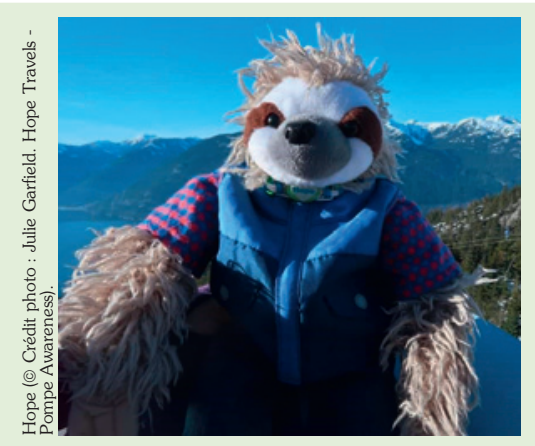

\section{LIENS D'INTÉR̂ET}

L'auteur déclare n'avoir aucun lien d'intérêt concernant les données publiées dans cet article.

\section{RÉFÉRENCES}

1. Elenga N, Verloes A, Mrsic Y, et al. Incidence of infantile Pompe disease in the Maroon population of French Guiana. BMJ Paediatr Open 2018; 2 : e000182.

2. Papadimas GK, Spengos K, Konstantinopoulou A, et al. Adult Pompe disease: clinical manifestations and outcome of the first Greek patients receiving enzyme replacement therapy. Clin Neurol Neurosurg 2011; 113 : 303-7.

3. Terzis G, Dimopoulos F, Papadimas GK, et al. Effect of aerobic and resistance exercise training on late-onset Pompe disease patients receiving enzyme replacement therapy. Mol Genet Metab $2011 ; 104: 279-83$

4. Terzis G, Krase A, Papadimas G, et al. Effects of exercise training during infusion on late-onset Pompe disease patients receiving enzyme replacement therapy. Mol Genet Metab 2012; 107 : 669-73.

5. Papadimas GK, Terzis G, Methenitis S, et al. Body composition analysis in late-onset Pompe disease. Mol Genet Metab $2011 ; 102: 41-3$.

6. Semplicini C, Letard P, De Antonio M, et al. Late-onset Pompe disease in France: molecular features and epidemiology from a nationwide study. J Inherit Metab Dis 2018 ; 41 : 937-46.

7. van der Beek NA, Hagemans ML, van der Ploeg AT, et al. The

Rasch-built Pompe-specific activity (R-PAct) scale. Neuromuscul

Disord 2013 ; 23 : 256-64.
8. Scheidegger O, Leupold D, Sauter R, et al. 36-Months follow-up assessment after cessation and resuming of enzyme replacement therapy in late onset Pompe disease: data from the Swiss Pompe registry. J Neurol 2018 ; 265 : 2783-8.

9. Stepien KM, Hendriksz CJ, Roberts M, et al. Observational clinical study of 22 adult-onset Pompe disease patients undergoing enzyme replacement therapy over 5years. Mol Genet Metab 2016 ; 117 : 413-8.

10. Lagalice L, Pichon J, Gougeon E, et al. Satellite cells fail to contribute to muscle repair but are functional in Pompe disease (glycogenosis type II). Acta Neuropathol Commun 2018; 6 : 116.

11. Slingerland NW, Polling JR, van Gelder CM, van der Ploeg AT, Bleyen I. Ptosis, extraocular motility disorder, and myopia as features of Pompe disease. Orbit 2011; 30 : 111-3.

12. Ravaglia S, Bini P, Garaghani KS, Danesino C. Ptosis in Pompe disease: common genetic background in infantile and adult series. J Neuroophthalmol 2010 ; 30 : 389-90.

13. Yanovitch TL, Banugaria SG, Proia AD, Kishnani PS. Clinical and histologic ocular findings in Pompe disease. J Pediatr Ophthalmol Strabismus 2010 ; 47 : 34-40.

14. Toussaint D, Danis P. Ocular histopathology in generalized glycogenosis (Pompe's disease). Arch Ophthalmol 1965; 73 : 342-9.

15. Armour SM, Nordin J, Puzzo F, et al. Safety and efficacy evaluation of investigational liver gene transfer for secretable GAA in the treatment of Pompe disease. Mol Genet Metab 2019; 126 : S24. 